\title{
Zinc Bioglasses Regulate Mineralisation in Human Dental Pulp Stem Cells
}

\section{Mei Huang 1, 2, ", Robert G. Hill², Simon C.F. Rawlinson'1}

1. Institute of Dentistry, Barts \& The London School of Medicine and Dentistry, Queen Mary University of London, UK

2. Institute of Dentistry, Dental Physical Sciences Unit, Barts \& The London School of Medicine and Dentistry, Queen Mary University of London, UK

${ }^{*}$ Correspondence to: Mei Huang, Institute of Dentistry, Barts \& The London School of Medicine and Dentistry, Queen Mary University of London, UK.

Email: mei.huang@qmul.ac.uk 


\begin{abstract}
Objective: A promising strategy in regenerative endodontics is the combination of human dental pulp stem cells (hDPSCs) with an appropriate biomaterial substrate. The effects of zinc and zinc containing bioactive glasses (ZnBGs) on hDPSCs have been characterised in this study.

Methods: ZnBGs were designed and produced. Then the odontogenic differentiation and mineralisation potential of hDPSCs upon $\mathrm{ZnBG}$ s treatment were investigated.

Results: Free $\mathrm{Zn}$ ions (0-5 ppm) enhanced proliferation and alkaline phosphatase (ALP) activity of hDPSCs. Further, ZnBGs conditioned medium (ZnBG-CM) increased the production and secretion of odontogenic markers: dentine sialophosphoprotein (DSPP), dentine matrix protein 1 (DMP-1). In addition, we identified that mRNA expression of the osteogenic markers RUNX2, OCN, BSP, $B M P-2, M E P E$ and $O N$ was increased following treatment with ZnBG-CM. Long term treatment with $\mathrm{ZnBG}-\mathrm{CM}$ increases the formation rate of mineralised nodules (similar to hydroxyapatite, $\mathrm{Ca}: \mathrm{P}=1.6)$, as confirmed by scanning electron microscopy combined with energy dispersive X-ray spectroscopy (SEM-EDX). Lastly, the administration of ZnBG-CM induces VEGF expression.
\end{abstract}

Significant: These findings implicate that ZnBG would be beneficial in regenerative endodontics and could influence the way present $\mathrm{Zn}$ containing clinical products are used. 


\section{Zinc Bioglasses Regulate Mineralisation in Human Dental Pulp Stem Cells}

\section{Introduction}

The high incidence of tooth decay and tooth loss combined with the limited regeneration capacities of dental tissues necessitates the development of novel strategies for dental practices [1]. Human dental pulp stem cells (hDPSCs) are a mesenchymal stem cell (MSC) population [2, 3]. Analogous to MSCs derived from bone marrow (BMSCs), hDPSCs can form alizarin red-positive mineralised deposits, physiologically similar to hydroxyapatite (HA) [2, 4]. Thus, experiments have been conducted to evaluate the value of hDPSCs in the repair of bone defects [5-7]. However, numerous studies have demonstrated that hDPSCs have a greater ability to differentiate into odontoblasts compared to BMSCs [2, 8]. Co-transplanting cultured hDSPCs with HA/TCP particles, into immunocompromised mice, led to the vascularisation of a fibrous pulp tissue surrounded by a layer of odontoblast-like cells, cells had extended tubular structures that resembling a dentine-like structure [2, 8]. In contrast, a lamellar bone structure with associated adipose tissue was formed by human BMSCs under the same experimental condition [2]. These findings clearly demonstrate distinct tissue regeneration capacities of hDPSCs and BMSCs, which appear to have maintained a level of epigenetic memory of their tissue origin [2]. Somewhat unsurprisingly then, hDPSCs is the preferred derivation of cells for dental tissue engineering.

The fundamental concept in hard tissue engineering lies in combining a scaffold with living cells to repair damaged tissue [9]. Scaffolds provide a suitable environment for cell attachment, proliferation, differentiation and to promote biomineralisation [10]. A significant challenge regarding scaffold usage is the choice of a suitable material. A broad range of materials, natural or synthetic polymers and inorganics, have been designed as scaffolds for dentin-pulp regeneration [11]. Bioactive glasses (BGs) are a promising choice for repair and 
reconstruction of hard tissues, such as bone and dentine $[12,13]$. BGs can form hydroxyapatite (HA) quickly, is biocompatible and can deliver 'therapeutic ions' $[12,14]$.

In dental practice, the use of zinc $(Z n)$ is mostly favoured owing to its bacteriostatic and cariostatic properties [15]. For example, Znoxide eugenol (ZOE), Zn oxyphosphate (ZOP), and Zn polycarboxylate (ZPC) are widely used. However, despite the clinical advantages, the dentinogenic benefits of $\mathrm{Zn}$ have yet to be determined. Accordingly, we have focused on $\mathrm{Zn}$ and $\mathrm{Zn}$ containing BGs (ZnBGs) to characterise their effects on hDPSCs.

\section{Materials and methods}

\subsection{Bioactive glass synthesis and characterisation}

ZnBGs (compositions are shown in Fig.2A) were synthesised by the meltquench route [16]. Glass frits were grounded and sieved to powders $\leq 38 \mu \mathrm{m}$. The particle size and amorphous nature was confirmed by master particle size analyser and X-ray diffraction (XRD). ZnBGs powders were characterised as described previously [17]. Briefly, $75 \mathrm{mg}$ of BGs powder $(\leq 38 \mu \mathrm{m})$ was added to $50 \mathrm{ml}$ Tris buffer and incubated on an orbital shaker (60 rpm at $37^{\circ} \mathrm{C}$ ). Post shaking, the $\mathrm{pH}$ values were recorded. The solution was filtered, diluted by a factor of ten in distilled $\mathrm{H}_{2} \mathrm{O}$ and acidified with $69 \%$ nitric acid (final concentration $2 \%$ ) prior to determining the elemental concentrations of calcium $(\mathrm{Ca})$, phosphorus $(\mathrm{P})$, silica $(\mathrm{Si})$, and zinc $(\mathrm{Zn})$ using an inductively coupled plasma-optical emission spectrometer (ICP-OES). The residual powders were dried overnight and analysed to evaluate the extent of apatite formation using $\mathrm{XRD}$, and Fourier transform infrared spectroscopy (FTIR).

\subsection{Cell culture}

Human dental pulp stem cells (hDPSCs, Lonza) were cultured in Dulbecco's Modified Eagle Medium (DMEM, Lonza) supplemented with $10 \%$ fetal calf 
serum (FCS) and antibiotics (10U/L penicillin and 100mg/L streptomycin) in a humidified atmosphere with $10 \% \mathrm{CO}_{2}$ at $37^{\circ} \mathrm{C}$.

Free ionic $\mathrm{Zn}(0.5,1,5,10$ and $20 \mathrm{ppm})$ was added as $\mathrm{ZnCl}_{2}$. $\mathrm{ZnBG}$ conditioned medium (ZnBG-CM) was prepared by immersing $75 \mathrm{mg}$ of glass powders in $50 \mathrm{ml}$ DMEM for set periods of time. Before being added to the cells, ZnBG-CM was filter-sterilized, serum and antibiotics were added and equilibrated to $37^{\circ} \mathrm{C}$, $10 \% \mathrm{CO}_{2}$ in an incubator.

Cells between passages 3-5 were used in all experiments. For cell proliferation, MTT and ALP activity assays, 1000 cells/well were seeded in 96-well plates and cultured for $1,4,7$ and 10 days. $1 \times 10^{5}$ cells/dish and $2 \times 10^{4}$ cells/well were placed in $10 \mathrm{~cm}$ dishes and 6-well plate for Western blot and qPCR studies, respectively. $5 \times 10^{3}$ cells were seeded onto sterilised coverslips and placed in 12-well plates for immunocytochemistry. 24-wells plate with $5 \times 10^{3}$ cells/well was used for the mineralisation study.

\subsection{MTT activity assay}

The MTT activity assay reflects mitochondrial activity-an indication of viable cells. After rinsing twice with PBS, $50 \mu$ l of MTT solution ( $5 \mathrm{mg} / \mathrm{ml}$ MTT in PBS) was added to cell cuture and incubated for $4 \mathrm{hrs}$ at $37^{\circ} \mathrm{C}$. After this, medium was removed and the formazan was solubilised in $100 \mu l$ dimethyl sulfoxide (DMSO). The absorbance of the solubilised formazan supernatant was measured at 560nm.

\subsection{Quantitative assay of ALP activity}

Alkaline phosphatase (ALP) activity was measured by adding $100 \mu$ l of ALP reaction solution (20mg 4-Nitropheyl-phosphate disodium salt hexahydrate tablet dissolved in $8 \mathrm{ml}$ Tris buffer solution ( $\mathrm{pH}=9.5$ ) containing $15 \mu \mathrm{l}$ of $2 \mathrm{M} \mathrm{MgCl}_{2}$ ) to cell lysate, incubated in the dark at $37^{\circ} \mathrm{C}$ for $1 \mathrm{hr}$. Afterwards, the reaction was stopped by adding $50 \mu \mathrm{l}$ of $1 \mathrm{M} \mathrm{NaOH}$. The absorbance of the supernatant was measured at $405 \mathrm{~nm}$ and ALP activity was calculated according to a standard curve (Fig.S1). 


\subsection{Crystal violet staining}

Cells were fixed with $10 \%$ neutral buffered formalin for 15 min, rinsed twice with PBS and stained in $0.5 \%(\mathrm{w} / \mathrm{v})$ crystal violet for $15 \mathrm{~min}$ at room temperature. Before visualising, the unbound stains were removed by extensive washing in PBS.

\subsection{Western Blot analysis}

Cell pellets were re-suspended in RIPA buffer (containing 1\% Triton X-100 and $1 \%$ cocktail proteinase inhibitors) and the protein content measured using DC $\mathrm{TM}$ Protein Assay. Intracellular expression of dentin sialophosphoprotein (DSPP), dentin matrix acidic phosphoprotein 1 (DMP-1) and vascular endothelial growth factor (VEGF) was evaluated by Western blotting using indicated antibodies (Table.A.1) on a PVDF membrane. Glyceraldehyde 3-phosphate dehydrogenase (GAPDH) was used as the loading control. Cell culture supernatant was also collected and analysed for DSPP and DMP-1 secretion, Coomassie blue was used to visualise sample separation. The band densities were quantified by ImageJ software.

\subsection{Immunocytochemistry}

4\% paraformaldehyde in PBS was used to fix the cells. Subsequently, cells were permeablised with $0.1 \%$ Triton $X-100$ for 10 min, and incubated with $10 \%$ goat serum for $30 \mathrm{~min}$ to block nonspecific binding. Following $1 \mathrm{hr}$ incubation in primary antibody (Table.S1) at room temperature, cells were washed and incubated with secondary antibody for $1 \mathrm{hr}$ and counter stained with DAPI. Finally, the cells were mounted on slides and viewed under confocal microscopy (LSM710, Zeiss). The images were then analysed with ImageJ software.

\subsection{Quantitative real-time polymerase chain reaction-qPCR}

Message RNA (mRNA) was isolated from cells with Dynabeads ${ }^{\circledR}$ (Thermo Fisher Scientific) and reverse-transcribed into cDNA using Transcriptor cDNA Synthesis Kit (Roche). The forward and reverse primers used for PCR were 
designed by Roche universal probe library according to the complementary DNA sequences available in GenBank (Table.S2). GPCR amplifications were performed on LightCycler 480 qPCR system (Roche) using LightCycler ${ }^{\circledR} 480$ SYBR Green I Master. For each PCR reaction, $2 \mu \mathrm{l}$ of 10 fold-diluted cDNA was used for amplification of the target genes. The thermal cycling conditions were as follows: $95^{\circ} \mathrm{C}$ for $5 \mathrm{~min}$ and $55 \mathrm{cycles}$ of $95^{\circ} \mathrm{C}$ for $10 \mathrm{~s}, 60^{\circ} \mathrm{C}$ for $6 \mathrm{~s}, 72^{\circ} \mathrm{C}$ for $6 \mathrm{~s}, 76^{\circ} \mathrm{C}$ for $1 \mathrm{~s}$. Relative expression levels of runt-related transcription factor 2 (RUNX2), osteonectin (ON), osteocalcin (OCN), bone sialoprotein (BSP), matrix extracellular phosphoglycoprotein (MEPE), and bone morphogenetic protein 2 (BMP-2) were determined with reference to GAPDH.

\subsection{Alizarin Red S staining}

After 3, 4 and 5 weeks culture, cells were fixed in $4 \%$ glutaraldehyde for $30 \mathrm{~min}$ and stained with $2 \%$ Alizarin Red $S$ (Sigma-Aldrich) for $1 \mathrm{hr}$ at room temperature. After three washes in $\mathrm{dH}_{2} \mathrm{O}$ to remove unbound stain, samples were air-dried and photographed. For quantification, the bound stain was extracted with $10 \%$ cetylpyridinum chloride, and the absorbance of solution was measured at $560 \mathrm{~nm}$.

\subsection{Scanning electron microscope (SEM)}

Cells were fixed in $2.5 \%$ glutaraldehyde for $1 \mathrm{hr}$ and dehydrated in graded ethanol $(50 \%, 70 \%, 80 \%, 90 \%, 95 \%$, and $100 \%)$, followed by critical-point drying with hexamethyldisilazane. The samples were coated with carbonate and viewed using SEM with an energy-dispersive X-ray (EDX) detector operated from $10-20 \mathrm{kV}$ to scan and analyse mineralised nodule formation and composition.

\subsection{Statistical analysis}

Statistical analysis was performed using GraphPad Prism 5. All data were collected from three independent experiments and presented as mean \pm standard deviation (SD). Differences were considered significant at $p<0.05$, 
determined using one-way ANOVA with Bonferroni's post hoc multiple comparison test.

\section{Results}

\subsection{Low dose $\mathrm{Zn}$ increases hDSPCs proliferation and ALP activity}

Cells were treated with $\mathrm{Zn}(0.5$ to $20 \mathrm{ppm})$ for $1,4,7$ and 10 days. $\mathrm{Zn}$ at low concentrations ( 0.5 and $1 \mathrm{ppm}$ ) showed no significant effect on cell proliferation (Fig.1A) as determined by MTT activity assays. Zn at $5 \mathrm{ppm}$ significantly increased $\left({ }^{*} p<0.05\right)$ cell proliferation. However, $\mathrm{Zn}$ at high doses (10 and 20 ppm) significantly reduced $\left({ }^{*} p<0.05\right)$ both cell proliferation. A significant increase $\left({ }^{*} p<0.05\right)$ in ALP activity is observed at low concentrations of $\mathrm{Zn}(0.5$ and $1 \mathrm{ppm}$ ) treatment for 7-10 days (Fig.1B). At 20 ppm, Zn reduced ALP activity (Fig.1B) due to increased cytotoxicity as visualised by crystal violet staining (Fig.1C).

\subsection{Characterisation of ZnBGs}

The amorphous nature of the ZnBGs was indicated by broad peaks in the XRD patterns (Fig.2B). Similar particle size distribution was confirmed by particle size analysis (Fig.S2A).

To evaluate bioactivity of the ZnBGs, immersion in Tris buffer was performed. An increase in $\mathrm{pH}$ was measured after immersion (Fig.S2B). The XRD and FTIR studies confirmed that $\mathrm{Zn}$ incorporation retards apatite formation in a dose-dependent manner (Fig.2C-F). Consistent with XRD findings, the FTIR data also suggested that $\mathrm{Zn}$ delays apatite formation (Fig.S3). ICP-OES analysis revealed rapid release of $\mathrm{P}$ and $\mathrm{Ca}$ ions from the glass within the first 6 hrs of immersion, these were then incorporated into apatite, hence the reduction of measurable levels. The concentration of $\mathrm{Si}$ increased continuously due to the continuous glass dissolution. Interestingly, no particular release pattern was observed for $\mathrm{Zn}$ (Fig. 2G-J), but the Zn concentration in all samples was less than $1 \mathrm{ppm}$. 


\subsection{ZnBG-CM regulates $\mathrm{hDPSC}$ behaviour and processing of DSPP and DMP-1}

hDPSCs were treated with ZnBG-CM. MTT assay demonstrated that ZnBG-CM has no significant effect on cell proliferation after 1 and 4 days of treatment. Cell proliferation was increased in $\mathrm{Zn} 1, \mathrm{Zn} 2$ and $\mathrm{Zn} 3 \mathrm{BG}-\mathrm{CM}$ treatment groups by day 7 and 10. Enhanced ALP activity was detected in hDPSCs following treatment with Zn3 BG-CM by day 1. However, by day 7 and 10, a significant increase $\left({ }^{*} p<0.05\right)$ in ALP activity was detected in all ZnBG-CM treatments when compared with the control group (Fig. 3B).

To further investigate the influence of $\mathrm{ZnBGs}$ on the odontogenic differentiation of hDPSCs, the regulation of two odontogenic markers - DSPP and DMP-1 was determined by Western blot and immunocytochemistry. As shown in Fig.3C, multiple bands were detected in DSPP and DMP-1 immunoblotting, that are indicative of protein processing as reported previously [18]. Densitometry analysis revealed that intracellular DSPP levels were significantly increased $\left({ }^{*} p<0.05\right)$ upon all ZnBG-CM treatment and intracellular DMP-1 levels were only enhanced in Zn2 and Zn3 BG-CM treatment for 7 days in culture. However, prolonged treatments that extend for 14 days suggested a reduction in intracellular DSPP and DMP-1 levels (Fig.3D). Immunoblotting of cell culture supernatant reflected the secretion of these two proteins (Fig.3E), where only DMP-1, but not DSPP, secretion is significantly increased $\left({ }^{*} p<0.05\right)$ following ZnBG-CM treatment (Fig.3F).

Immunocytochemistry and confocal microscopy analysis demonstrated that DSPP is localised to nucleus and perinuclear region (confirmed by co-staining with the Golgi marker GM130, Fig.4A and C). Further, DSPP localisation to Golgi was induced following ZnBG-CM treatment (Fig.4B and C). DMP-1 is shown to be localised in both cytosol and nucleus (Fig.4A) and no change in its localisation pattern is induced by ZnBG-CM treatment (Fig.S4).

\subsection{ZnBG induces mineralisation of hDPSCs}


The mineralisation potential of hDPSCs following ZnBG-CM treatment was assessed by Alizarin Red $S$ that stains $\mathrm{Ca}^{2+}$ deposits red (Fig.5A). Quantification of the staining demonstrated significant increases $\left({ }^{*} p<0.05\right)$ in the staining induced by treatment with Zn1, Zn2 and Zn3 BG-CM by week 3, 4 and 5 in comparison to the glass free control group. SEM scans revealed that the mineralised nodules generated by hDPSCs were between 50 and $100 \mu \mathrm{m}$ in diameter and were raised from the culture surface (Fig.5C). EDX spectra for calcium and phosphate content in the mineralised nodules indicated a ratio of Ca:P similar to that of hydroxyapatite ( 1.6, Fig.5C).

\subsection{ZnBG modulates mineralisation-associated gene expression}

Further regulation of mineralisation-related differentiation markers of hDPSCs was investigated by $\mathrm{qPCR}$. This analysis revealed up-regulation of $B S P$ and MEPE by day 7 and 14 following all conditions of ZnBG-CM treatment (Fig. 5G and $\mathrm{H}$ ). High expression levels of RUNX2 and BMP-2 were detected for $\mathrm{Zn} 1$, $Z n 2$ and $Z n 3$ groups but not $Z n 0$ group. Differential expression was observed for $\mathrm{ON}$, when compared to other groups, and $\mathrm{OCN}$ is increased only by groups $\mathrm{Zn} 0$ and $\mathrm{Zn} 3$ at day 7 . However, by day 14, the mRNA level of all the genes was up-regulated following $\mathrm{ZnBG}-\mathrm{CM}$ treatment irrespective of the $\mathrm{Zn}$ content (Fig.5D-I).

\subsection{ZnBG modulation of VEGF levels}

Following ZnBG-CM treatment immune blotting and densitometry analysis revealed that $Z n 1$ and $Z n 3$ groups clearly enhanced intracellular VEGF levels in hDPSCs after 7 days. Furthermore, by day 14 the expression of VEGF was significantly increased $\left({ }^{*} p<0.05\right)$ in all ZnBG-CM treatments (Fig $\left.5 \mathrm{~J}-\mathrm{K}\right)$.

\section{Discussion}

The use of $45 \mathrm{~S} 5 \AA$ BG in regenerative endodontics for pulp capping demonstrated the formation of a reparative dentine bridge $[19,20]$. The amorphous BG is generally more soluble and reactive than crystalline materials, 
such as calcium phosphate, thereby enhancing the bioactivity of the material. In addition, BGs can be manufactured to release 'therapeutic' ions, which may induce accelerated formation of mineralised tissues. As $\mathrm{Zn}$ is already widely used in dentistry, we have investigated the effect of ZnBGs on differentiation and mineralisation of hDPSCs.

Initially, the safe range of free $\mathrm{Zn}$ ion was accessed in cultures of hDPSCs. Cell proliferation and ALP activity assays suggested that low dose $\mathrm{Zn}$, from 0 to 5 ppm, is not cytotoxic and induces the proliferation and differentiation of hDPSCs. High concentrations (10 and 20ppm) are toxic (Fig. 1). These findings suggested that ZnBG might also be able to positively modulate hDPSCs odontogenic behaviour.

The influence of $\mathrm{Zn}$ on the bioactivity process and its role in BG structure has been extensively debated in literature [21]. ZnO can either act as a modifier leading to a decrease in surface area and pore size of the BG [22]. ZnO can also act as an intermediate oxide forming covalent links between adjacent $\mathrm{SiO}_{4}$ tetrahedra [23] to create a more stable glass structure. In this study, Zn was added to the BGs assuming that it behaves entirely as an intermediate oxide. The ZnBGs were successfully synthesised as amorphous structures. The bioactivity of these ZnBGs was quantified (assessed by apatite formation) when immersed in a relevant physiological fluid. [24]. XRD and FTIR findings both confirmed that $\mathrm{Zn}$ addition to $\mathrm{BG}$ delays the apatite formation in a dosedependent manner (Fig. 2). This agrees with previous findings, where delayed apatite formation was due to an increase in the network polymerisation with the incorporation of $\mathrm{Zn}$ [25].

To determine any biological effect, ZnBG-CMs were used in cell culture. At early time points ( 1 and 4 days), ZnBG-CMs showed no effect on cell proliferation, thereafter increased cell proliferation was observed by day 7 and 10 (Fig. 3). These findings indicate that these novel ZnBGs have no significant cytotoxic effects on hDPSCs, confirming the possible utilisation of these materials in tissue repair. The observed increase in ALP activity with ZnBG 
treatment over time indicates the differentiation potential of hDPSCs and confirmed by the subsequent increase in mineralisation (Fig. 3).

Protein levels of the odontogenic markers DSPP and DMP-1 were quantified to determine odontogenic differentiation of hDPSCs following ZnBG-CM treatment (Fig. 3). DSPP and DMP-1 are both involved in the nucleation and formation of HA during dentine formation [26]. Multiple bands were observed in DSPP and DMP-1 blotting, that reflect small functional cleavage products: dentine sialoprotein (DSP) and dentine phosphoprotein (DPP) [26]. Densitometry analysis revealed DSPP levels were increased by day 7 and reduced by day 14 . Meanwhile, immunocytochemistry and confocal microscopy analysis revealed DSPP localisation to Golgi was induced upon ZnBG-CM treatment (Fig. 4). This implies that ZnBG increases intracellular DSPP protein levels, and subsequently processed in Golgi as reported previously [18]. Increases in DMP1 levels and secretion are detected as two fragments: N-terminal $(37 \mathrm{kDa})$ and C-terminal fragments (57kDa) [26], was also observed following ZnBG-CM treatment (Fig.4E). However, no changes in DMP-1 cellular localisation were observed in response to ZnBG-CM treatment. These findings lend support to the idea that ZnBGs in part induce odontogenic differentiation of hPDSCs. PCR analysis also revealed increase in both $D S P P$ and $D M P-1$ mRNA expression along with osteogenic markers (RUNX2, BMP1, BSP, ON, OCN, and MEPE), which are crucial genes associated with critical roles in mineralisation [27-29]. These results are consistent with previous investigation showing that $\mathrm{Zn}$ added BG granules stimulate growth and osteogenic differentiation of MSCs via regulation of ALP activity and BSP expression [30]. Together, these findings suggest that ZnBGs can enhance differentiation of hDPSCs in a fashion similar to osteoblast differentiation. This in turn impacts on the mineralisation potential of hDPSCs, as observed in our studies by Alizarin Red S staining and SEMEDX (Fig. 5). Moreover, western blot analysis reveals that the expression of VEGF is increased following ZnBG-CM treatment. This is in agreement with a previous report that VEGF plays an essential role in the regulation of mineralisation by stimulating differentiation of MSCs [31]. VEGF has also been confirmed to promote odontogenic differentiation of hDPSCs in vitro [32, 33]. 
In a similar study, the stimulation of odontogenic differentiation of dental pulp cells following ZnBG incorporated cement treatment was reported. In addition, this ZnBG incorporated cement up-regulated the expression of VEGF and the increased the tubular structure in endothelial cells [34]. The findings in present study, in consistent with the previous investigation [34], clearly indicate that $\mathrm{ZnBGs}$ can promote the generation of mineralised nodules via up-regulation of VEGF in both osteogenic and odontogenic differentiation pathways in hDPSCs. It also raises the question, what effect are clinical treatments that contain $\mathrm{Zn}$ having on the local cells, and especially odontoblasts?

\section{Conclusion}

Stem cell-based regenerative endodontics is a new and exciting field that has the potential to transform the dental practice. In this study, novel zinc containing bioactive glasses were successfully designed and synthesised. Biological studies demonstrated activity of the odonto/osteogenic and VEGF pathways that might be the mechanism by which $\mathrm{Zn}$ induces the differentiation of hDPSCs. This study raises the intriguing possibility of using ZnBGs as therapeutic scaffolds in regenerative endodontics.

\section{Conflicts of Interest}

The authors declare no conflict of interest.

\section{Acknowledgements}

The authors would like to thank China Scholarship Council (CSC) for the joint PhD studentship program with Queen Mary University of London. The authors would like to thank BALM imaging facilities at Blizard Institute Queen Mary University of London. The authors declare no potential conflicts of interest with respect to the authorship and/or publication of this article.

\section{Reference:}


1. Malhotra, N. and K. Mala, Regenerative endodontics as a tissue engineering approach: past, current and future. Aust Endod J, 2012. 38(3): p. 137-48.

2. Gronthos, S., et al., Postnatal human dental pulp stem cells (DPSCs) in vitro and in vivo. Proceedings of the National Academy of Sciences, 2000. 97(25): p. 13625-13630.

3. Gronthos, S., et al., Stem Cell Properties of Human Dental Pulp Stem Cells. Journal of Dental Research, 2002. 81(8): p. 531-535.

4. Couble, M.-L., et al., Odontoblast Differentiation of Human Dental Pulp Cells in Explant Cultures. Calcified tissue international, 2000. 66(2): p. 129-138.

5. Zheng, Y., et al., Stem cells from deciduous tooth repair mandibular defect in swine. J Dent Res, 2009. 88(3): p. 249-54.

6. Liu, H.-C., et al., Reconstruction of Alveolar Bone Defects Using Bone Morphogenetic Protein 2 Mediated Rabbit Dental Pulp Stem Cells Seeded on Nano-Hydroxyapatite/Collagen/Poly(L-lactide). Tissue Engineering Part A, 2011. 17(19-20): p. 2417-2433.

7. Yamada, Y., et al., Promising Cell-Based Therapy for Bone Regeneration Using Stem Cells From Deciduous Teeth, Dental Pulp, and Bone Marrow. Cell Transplantation, 2011. 20(7): p. 1003-1013.

8. $\mathrm{Yu}, \mathrm{J}$., et al., Odontogenic capability: bone marrow stromal stem cells versus dental pulp stem cells. Biol Cell, 2007. 99(8): p. 465-74.

9. Langer, R. and J.P. Vacanti, Tissue engineering. Science, 1993. 260(5110): p. 920-6.

10. Bottino, M.C., et al., Bioactive nanofibrous scaffolds for regenerative endodontics. J Dent Res, 2013. 92(11): p. 963-9.

11. Khanna-Jain, R., et al., Osteogenic differentiation of human dental pulp stem cells on beta-tricalcium phosphate/poly (I-lactic acid/caprolactone) threedimensional scaffolds. J Tissue Eng, 2012. 3(1): p. 2041731412467998.

12. Hench, L., The story of Bioglass $®$. Journal of Materials Science: Materials in Medicine, 2006. 17(11): p. 967-978.

13. Qu, T. and X. Liu, Nano-Structured Gelatin/Bioactive Glass Hybrid Scaffolds for the Enhancement of Odontogenic Differentiation of Human Dental Pulp Stem Cells. J Mater Chem B Mater Biol Med, 2013. 1(37): p. 4764-4772.

14. Xynos, I.D., et al., Ionic Products of Bioactive Glass Dissolution Increase Proliferation of Human Osteoblasts and Induce Insulin-like Growth Factor II $m R N A$ Expression and Protein Synthesis. Biochemical and Biophysical Research Communications, 2000. 276(2): p. 461-465.

15. Tenovuo, J., et al., Release of cariostatic agents from a new buffering fluorideand xylitol-containing lozenge to human whole saliva in vivo. J Oral Rehabil, 1997. 24(5): p. 325-31.

16. Hench, L.L. and H.A. Paschall, Direct chemical bond of bioactive glass-ceramic materials to bone and muscle. Journal of Biomedical Materials Research, 1973. 7(3): p. 25-42.

17. Mneimne, M., et al., High phosphate content significantly increases apatite formation of fluoride-containing bioactive glasses. Acta Biomater, 2011. 7(4): p. 1827-34.

18. Huang, M., R.G. Hill, and S.C. Rawlinson, Strontium (Sr) elicits odontogenic differentiation of human dental pulp stem cells (hDPSCs): A therapeutic role for Sr in dentine repair? Acta Biomater, 2016. 27(16): p. 30199-4.

19. Oguntebi, B., A. Clark, and J. Wilson, Pulp capping with Bioglass and autologous demineralized dentin in miniature swine. J Dent Res, 1993. 72(2): $p$. 484-9. 
20. Oguntebi, B.R., et al., Quantitative assessment of dentin bridge formation following pulp-capping in miniature swine. Journal of Endodontics, 1995. 21(2): p. 79-82.

21. Balasubramanian, P., et al., Zinc-containing bioactive glasses for bone regeneration, dental and orthopedic applications, in Biomedical glasses. 2015.

22. Miola, M., et al., Electrophoretic Deposition of Chitosan/45S5 Bioactive Glass Composite Coatings Doped with $\mathrm{Zn}$ and Sr. Frontiers in Bioengineering and Biotechnology, 2015. 3: p. 159.

23. Lusvardi, G., et al., Synthesis, Characterization, and Molecular Dynamics Simulation Of Na2O-CaO-SiO2-ZnO Glasses. The Journal of Physical Chemistry B, 2002. 106(38): p. 9753-9760.

24. Kokubo, T. and $\mathrm{H}$. Takadama, How useful is SBF in predicting in vivo bone bioactivity? Biomaterials, 2006. 27(15): p. 2907-15.

25. Mohammed, N.R., et al., Physical chemical effects of zinc on in vitro enamel demineralization. J Dent, 2014. 42(9): p. 1096-104.

26. Suzuki S, H.N., Nishimura F, Kulkarni AB., Dentin sialophosphoprotein and dentin matrix protein-1: Two highly phosphorylated proteins in mineralized tissues. Archives of Oral Biology, 2012. 57(9): p. 1165-1175.

27. Chen, S., et al., Runx2, osx, and dspp in tooth development. J Dent Res, 2009. 88(10): p. 904-9.

28. Casagrande, L., et al., Dentin-derived BMP-2 and odontoblast differentiation. J Dent Res, 2010. 89(6): p. 603-8.

29. Goldberg, M., et al., Dentin Structure, Composition and Mineralization. Front Biosci, 2011. 1(3): p. 711-735.

30. Oh, S.A., et al., Effects on growth and osteogenic differentiation of mesenchymal stem cells by the zinc-added sol-gel bioactive glass granules. J Tissue Eng, 2011. 2010: p. 475260.

31. Mayer, H., et al., Vascular endothelial growth factor (VEGF-A) expression in human mesenchymal stem cells: Autocrine and paracrine role on osteoblastic and endothelial differentiation. Journal of Cellular Biochemistry, 2005. 95(4): p. 827-839.

32. Matsushita, K., et al., The role of vascular endothelial growth factor in human dental pulp cells: induction of chemotaxis, proliferation, and differentiation and activation of the AP-1-dependent signaling pathway. J Dent Res, 2000. 79(8): p. 1596-603.

33. Zhang, W., et al., Odontogenic differentiation of vascular endothelial growth factor-transfected human dental pulp stem cells in vitro. Mol Med Rep, 2014. 10(4): p. 1899-906.

34. Zhang, J., et al., Effects of bioactive cements incorporating zinc-bioglass nanoparticles on odontogenic and angiogenic potential of human dental pulp cells. J Biomater Appl, 2015. 29(7): p. 954-64.

\section{Figure Legends:}

Figure 1. Effects of free $\mathrm{Zn}$ ion on hDPSCs. (A): MTT activity of hDPSCs treated with Zn (020ppm). Data is presented as fold change upon comparison with $\mathrm{Zn}$ Oppm group at day 1. (B): ALP activity of hDPSCs following $\mathrm{Zn}(0-20 \mathrm{ppm})$ treatment. The ALP activity was measured in each group and normalised to cell number. (C): Crystal violet staining of hDPSCs after 10 days exposure to $\mathrm{Zn}(0-20 \mathrm{ppm})$. Scale bar $=100 \mu \mathrm{m}$. All the data are expressed as mean \pm SD from 
three independent experiments. ${ }^{*}$ indicates significance value of $\mathrm{p}<0.05$ when compared to $\mathrm{Zn}$ Oppm group.

Figure 2. Characterisation of ZnBGs. (A): ZnBG compositions, the network connectivity for all the ZnBGs are fixed at 2.1. (B): XRD patterns of all unreacted BGs confirm amorphous nature. (C) - (F): XRD pattern of ZnBGs after Tris buffer dissolution from 0 to $168 \mathrm{hrs}$, two peaks at 26 and $322 \theta^{\circ}$ indicate apatite formation. (G) - (J): ICP-OES analysis of ZnBGs after immersion in Tris buffer for $168 \mathrm{hrs}$. All ionic (P, Ca, $\mathrm{Si}$, and $\mathrm{Zn})$ data in ppm was plotted as a function of time.

Figure 3. Effects of ZnBG on odontogenic differentiation. (A): Cell proliferation of hDPSCs treated with ZnBG-CMs was assessed by MTT activity assay. Data is presented as a fold change compared to negative control group at day 1 (B): ALP activity of hDPSCs following ZnBG-CMs treatment. The ALP activity was measured in each group and normalised to cell number. (C): Western blot analysis of intracellular DSPP and DMP-1 expression, GAPDH expression was used to determine the loading control. Lane 1-5: Negative control (NC), Zn0, Zn1, Zn2 and Zn3 BG$\mathrm{CM}$, respectively. (D): Relative intensities of intracellular DSPP and DMP-1 to GAPDH were obtained, and then compared to NC group to determine the fold change. (E): Western blot analysis for secreted DSPP and DMP-1. Coomassie blue staining was used as loading control. The lanes setup is same as in (C). (F): Relative intensities of secreted extra cellular DSPP and DMP-1 were obtained by Coomassie blue staining, and then compared to NC group to determine fold changes. All data were expressed as mean $\pm \mathrm{SD}$ from three independent experiments, ${ }^{*}$ indicates significance of $\mathrm{p}<0.05$.

Figure 4. ZnBGs regulate DSPP and DMP-1 localisation in hDPSCs. (A): Representative images show staining of DSPP and DMP-1 in red for hDPSCs. Scale bar $=8 \mu \mathrm{m}$. (B): Representative images of DSPP and GM130 (Golgi marker) staining in hDPSCs following ZnBG-CM treatment. Images show DSPP (green) and Golgi (red) and cell nuclei (blue). Scale bar $=30 \mu \mathrm{m}$. (C): The colocalisation of DSPP (green) and GM130 (red) in hDPSCs was quantified and represented in bar chart after treated with ZnBG-CMs Scale bar $=8 \mu \mathrm{m}$. Data was expressed as mean \pm SD from three independent experiments, and ${ }^{*}$ denotes $\mathrm{p}<0.05$ significance where indicated.

Figure 5. ZnBG-CM regulates mineralisation and osteogenic differentiation of hDPSCs. (A): Representative images show Alizarin Red $\mathrm{S}$ staining indicating mineralisation (in red colour) of hDPSCs. (B): Quantification of Alizarin Red S staining in hDPSCs was presented as a fold change compared to negative control (NC) group. (C): SEM-EDX analysis of mineralised nodules generated in hDPSCs. (D)-(I): qPCR analysis of RUNX2, ON, OCN, BSP, MEPE and BMP-2 expression in DPSCs following ZnBG-CM treatment for 7 and 14 days. The expression level of each gene was normalized to GAPDH (reference gene). Fold change was determined by comparing to NC group at day 7. (J): Western blot analysis of intracellular VEGF expression, and GAPDH levels were determined for loading control. Lane1-5: NC, Zn0, Zn1, Zn2 and Zn3BGCM. (D): Relative intensities of VEGF in reference to GAPDH were determined and expressed as fold change in comparison to, NC. All the data were expressed as mean \pm SD from three independent experiments, and the significance is denoted as ${ }^{*} p<0.05,{ }^{*} p<0.01$ where indicated. 
(A)

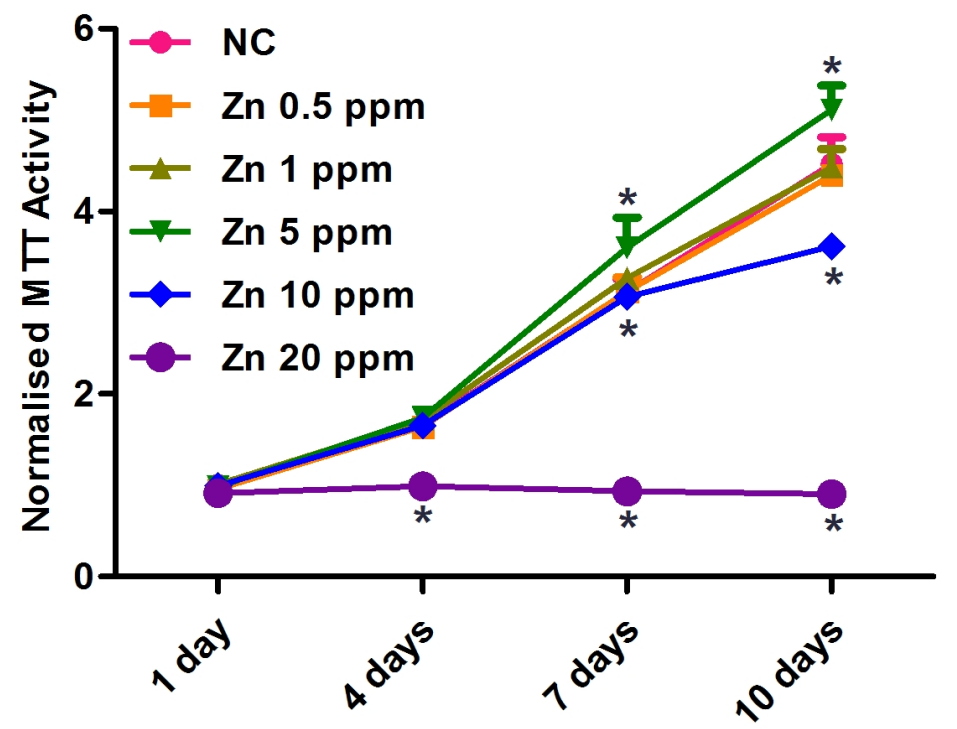

(B)

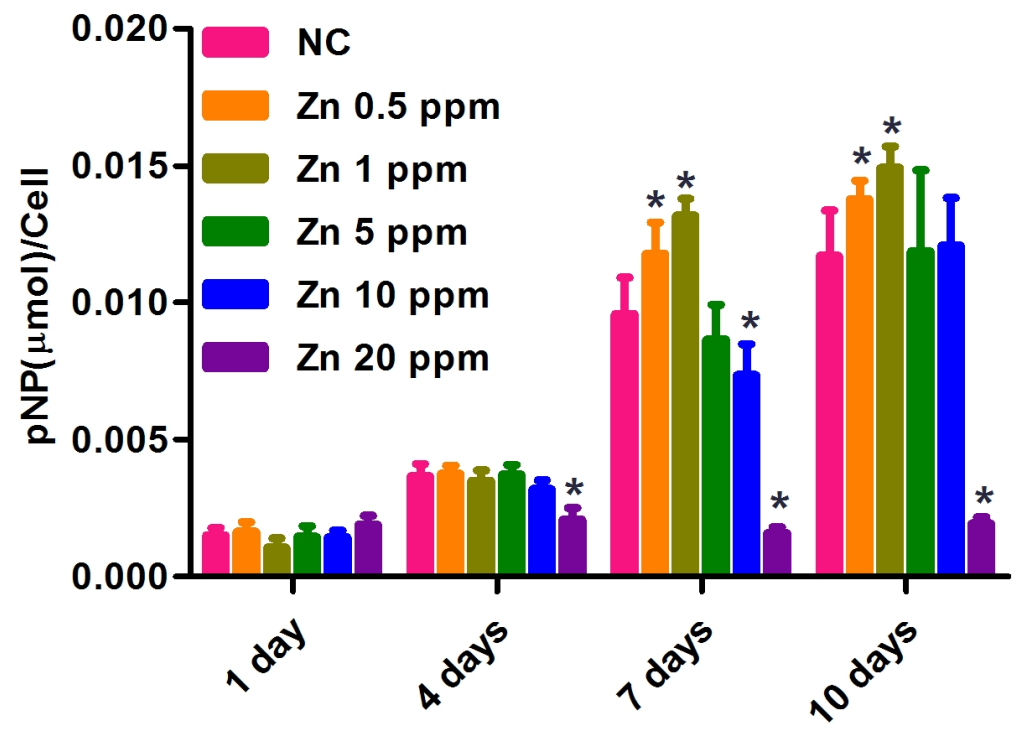

(C)

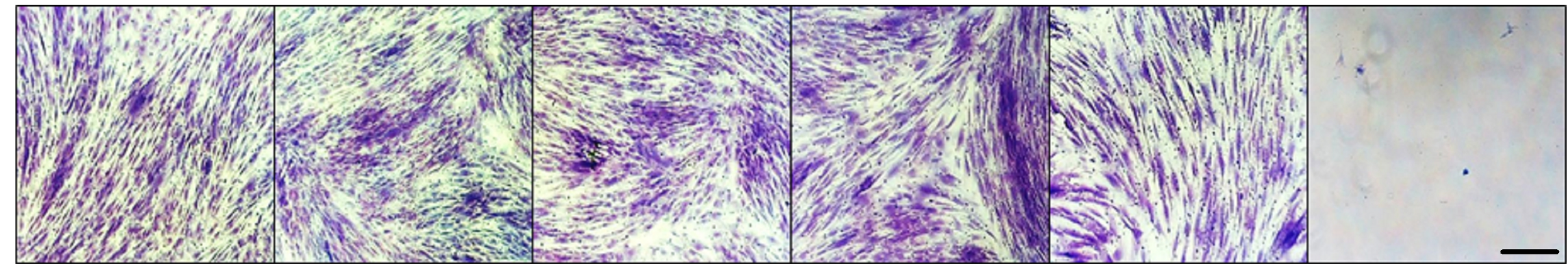


(B)

\begin{tabular}{|c|c|c|c|c|c|}
\hline Glass & $\mathbf{S i O}_{2}$ & $\mathbf{N a}_{2} \mathbf{O}$ & $\mathrm{CaO}$ & $\mathbf{P}_{\mathbf{2}} \mathbf{O}_{\mathbf{5}}$ & $\mathbf{Z n O}$ \\
\hline $\mathbf{Z n 0}$ & 38.5 & 26.2 & 29 & 6.3 & 0.0 \\
\hline $\mathbf{Z n 1}$ & 37.0 & 26.5 & 29.2 & 6.3 & 1.0 \\
\hline $\mathbf{Z n 2}$ & 35.7 & 26.7 & 29.4 & 6.2 & 2.0 \\
\hline $\mathbf{Z n 3}$ & 34.3 & 27.0 & 29.6 & 6.1 & 3.0 \\
\hline
\end{tabular}

ZnBG compositions in mol\% with a fixed network connectivity of 2.1 .

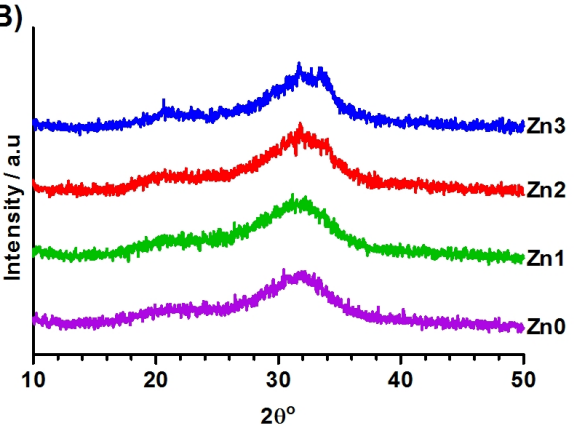

(C)

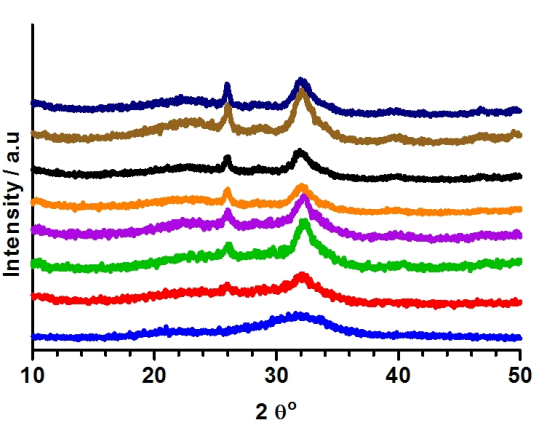

(E)

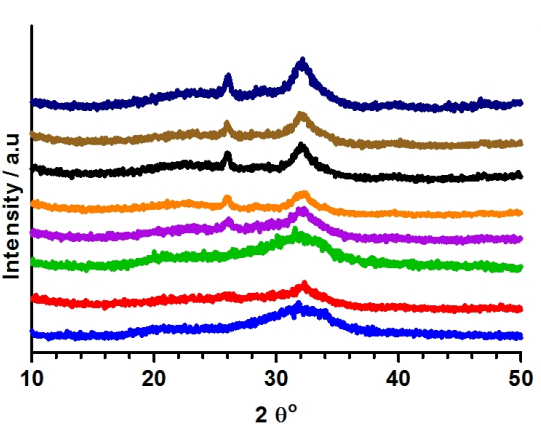

(G)

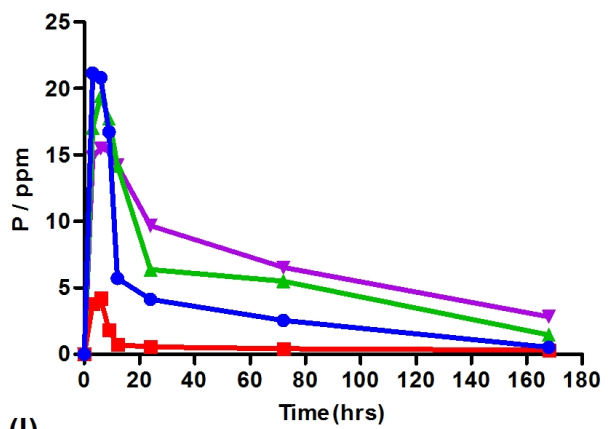

(I)

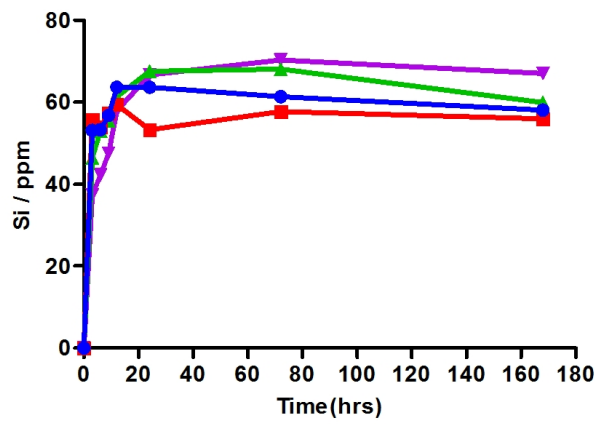

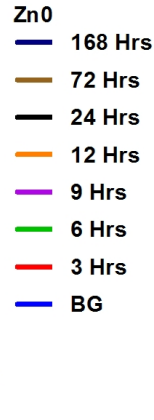

$\stackrel{\mathrm{Zn} 2}{-} 168 \mathrm{Hrs}$

$72 \mathrm{Hrs}$

- $24 \mathrm{Hrs}$

$12 \mathrm{Hrs}$

$9 \mathrm{Hrs}$

$6 \mathrm{Hrs}$

- $3 \mathrm{Hrs}$

- BG

$\rightarrow$ Zno

$-\mathrm{Zn} 1$

$\pm \mathrm{Zn} 2$

$\rightarrow \mathrm{Zn} 3$

$\rightarrow$ Zno

$\rightarrow \mathrm{Zn} 1$

$\pm \mathrm{Zn} 2$

$+\mathrm{Zn} 3$
(D)

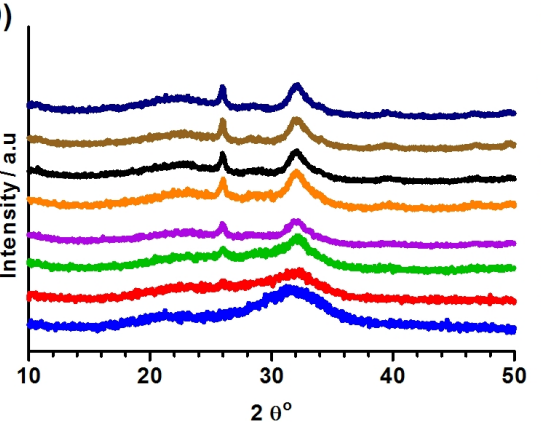

(F)

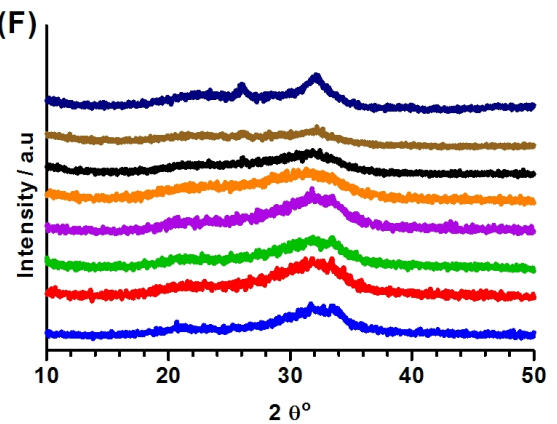

(H)

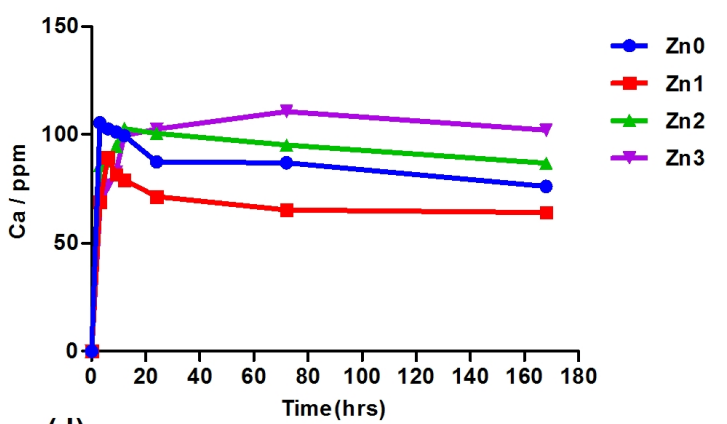

(J)

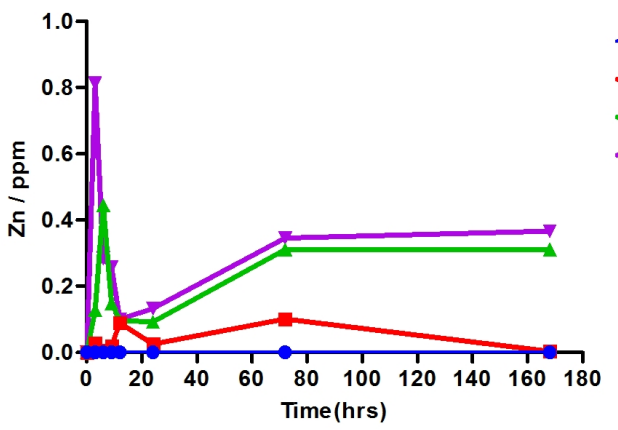

Zn1

- $168 \mathrm{Hrs}$

$72 \mathrm{Hrs}$

- $24 \mathrm{Hrs}$

$12 \mathrm{Hrs}$

- $9 \mathrm{Hrs}$

$6 \mathrm{Hrs}$

- $3 \mathrm{Hrs}$

- BG
$72 \mathrm{Hrs}$

- $24 \mathrm{Hrs}$

$12 \mathrm{Hrs}$

- $9 \mathrm{Hrs}$

- $6 \mathrm{Hrs}$

- 3 Hrs

- BG 
(A)

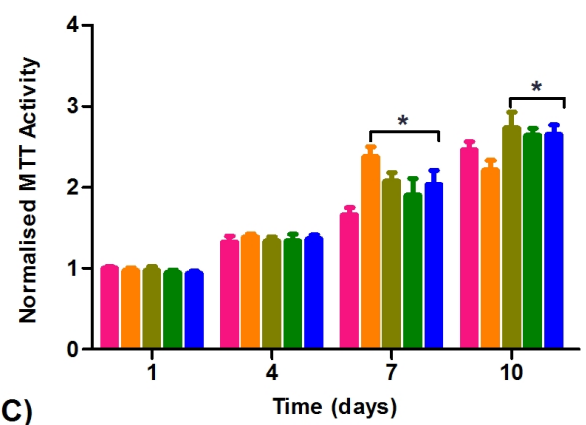

(C)

Whole cell lys ate

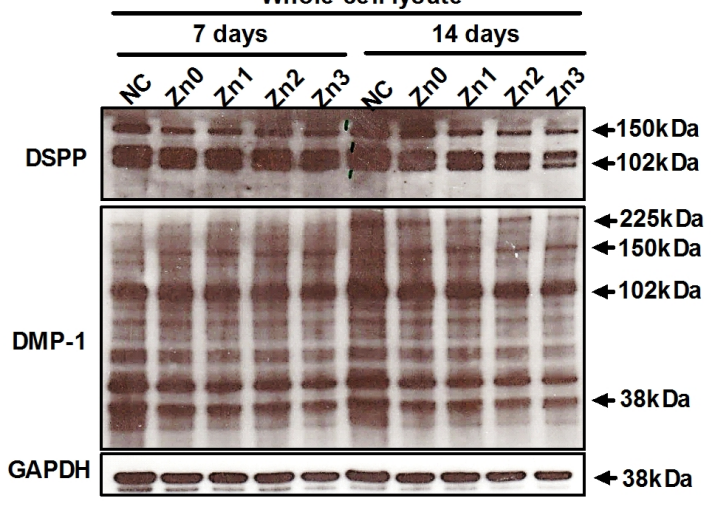

(E)

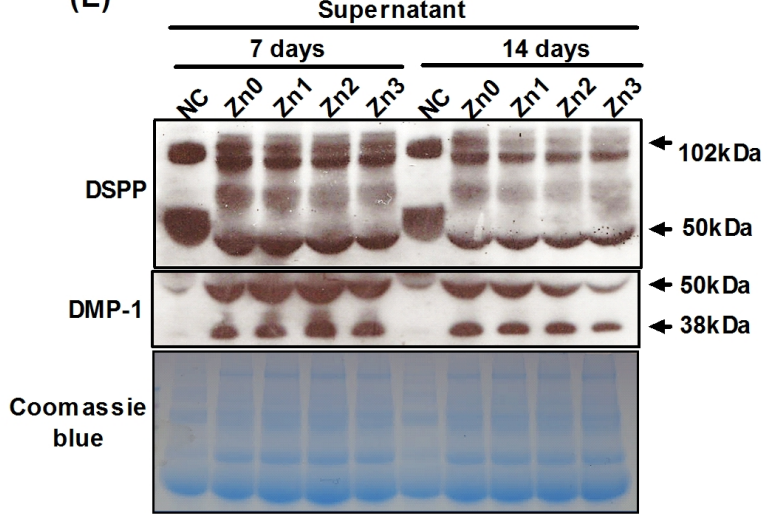

(B)

ALP activity
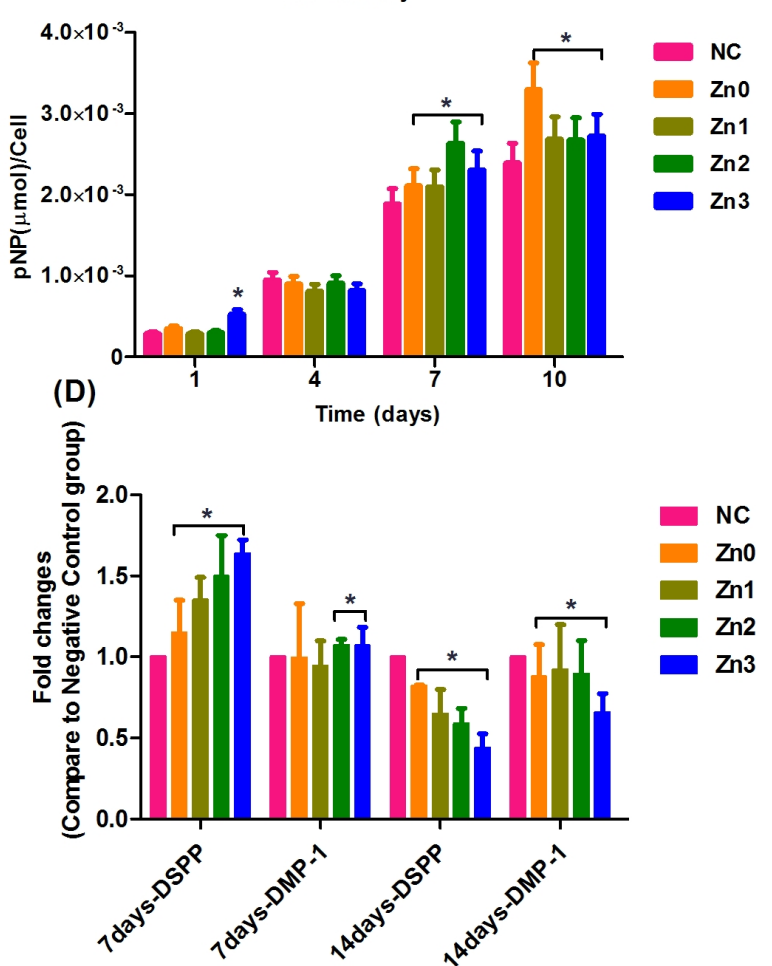

(F)

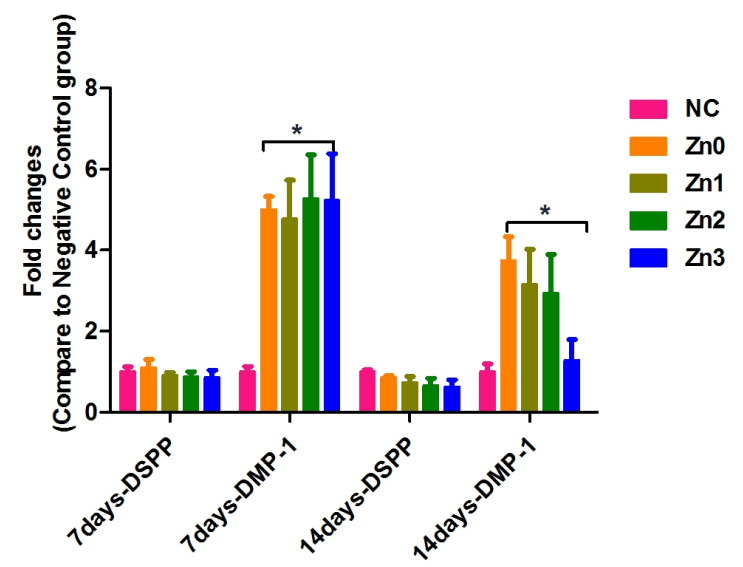


(A)

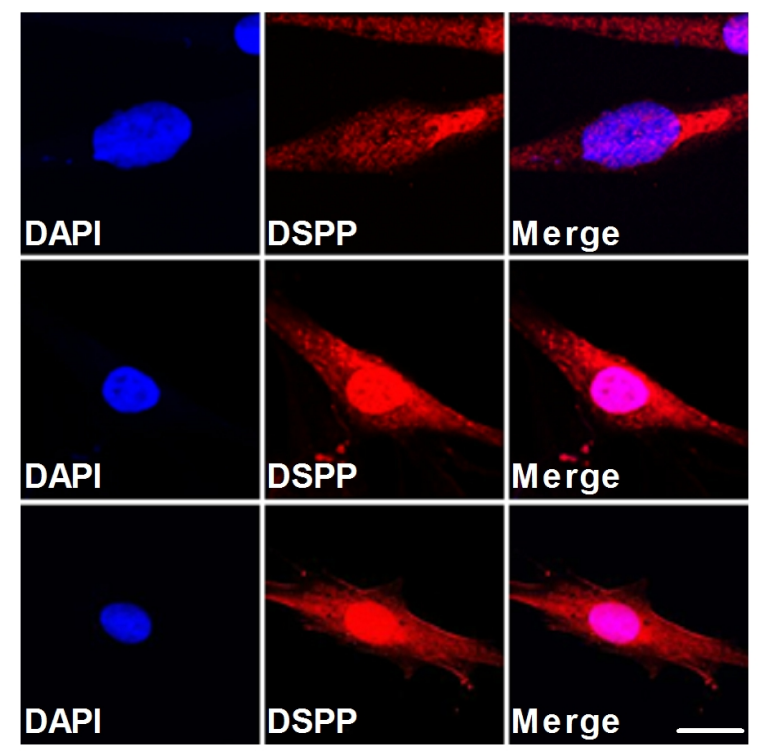

(C)

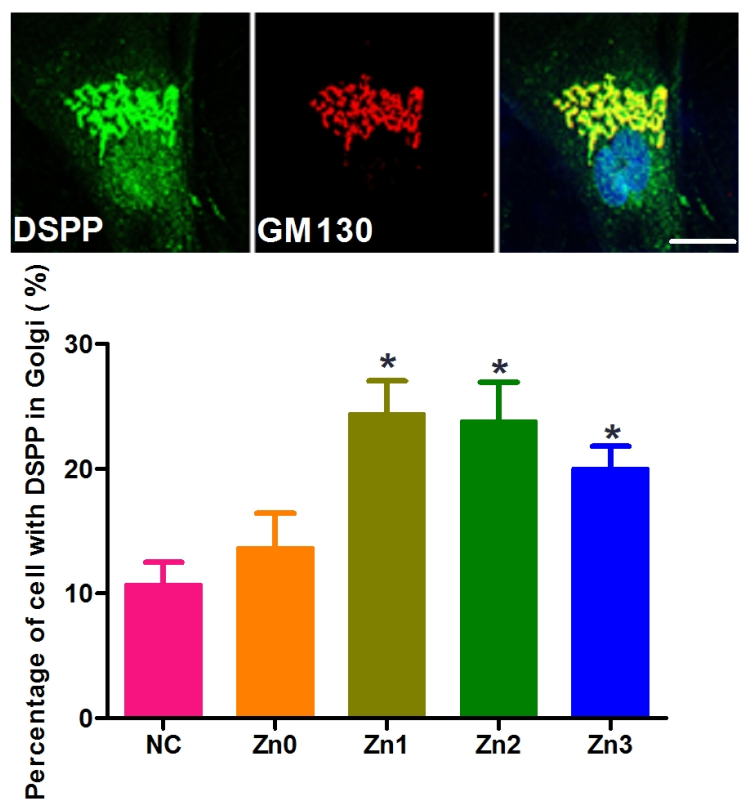

(B) NC Zno Zn1
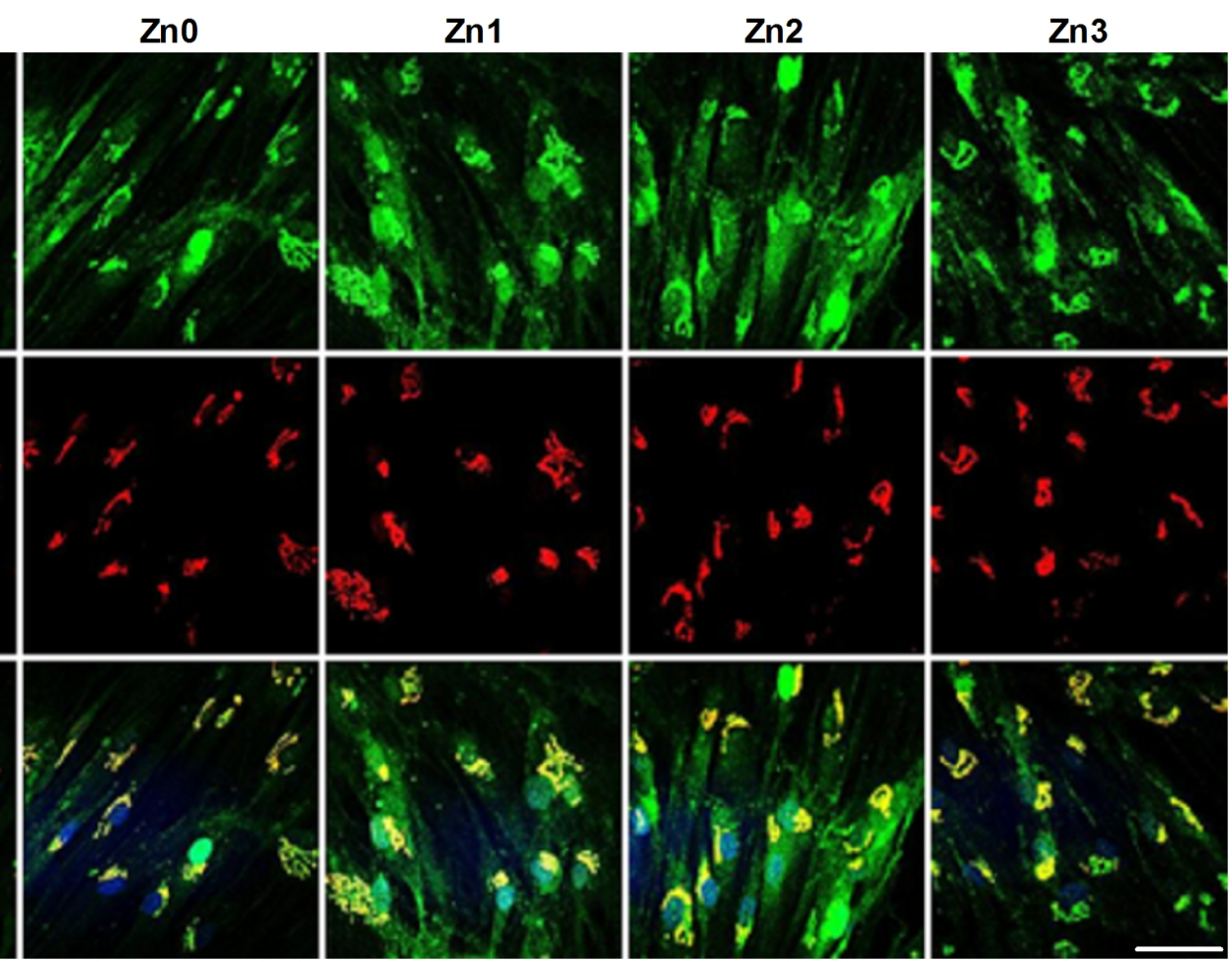


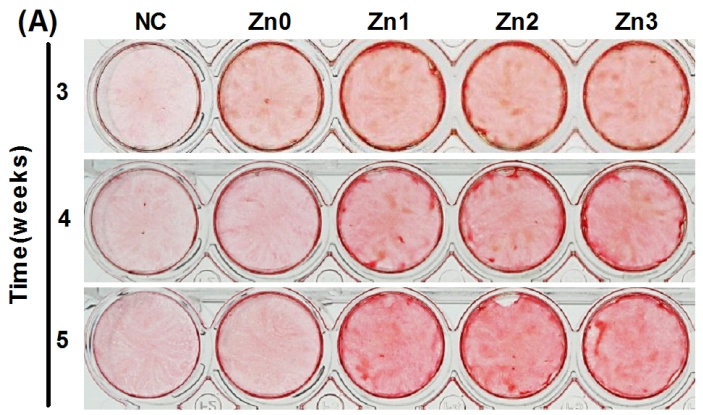

(B)

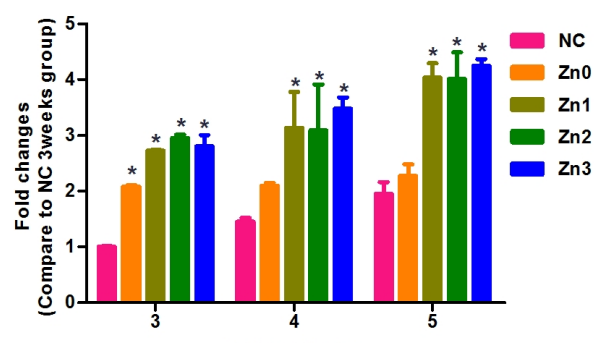

(C)
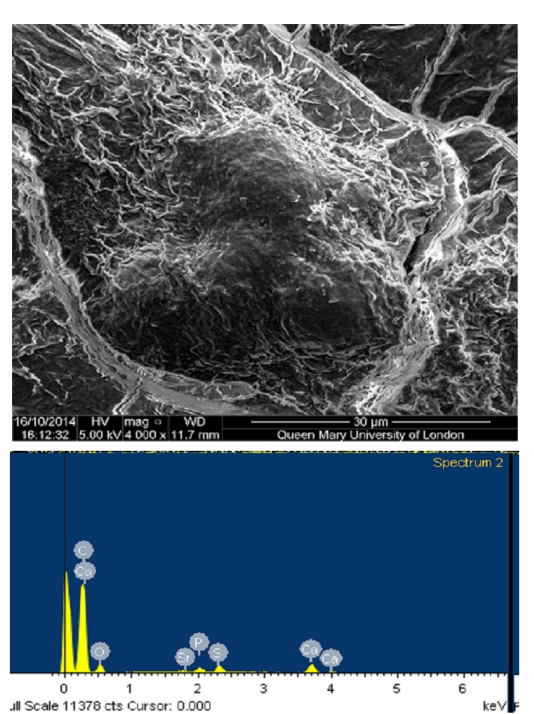

(D)

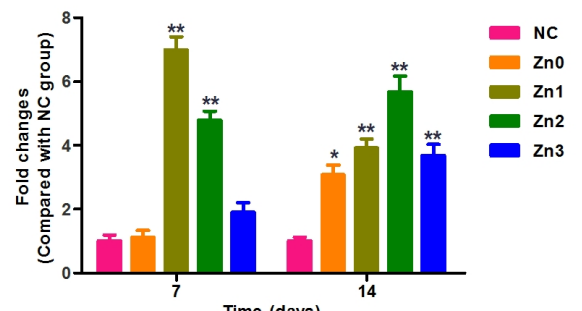

(F)

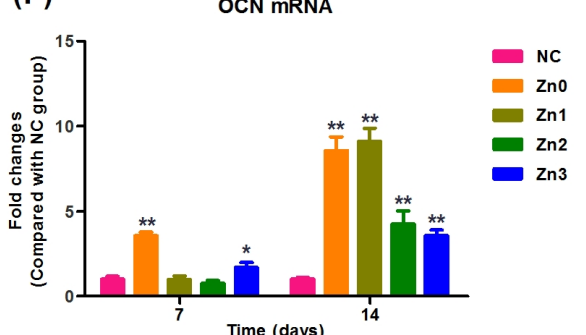

(H) BSP MRNA

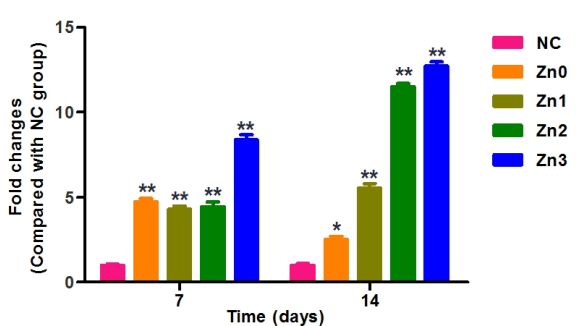

(J)

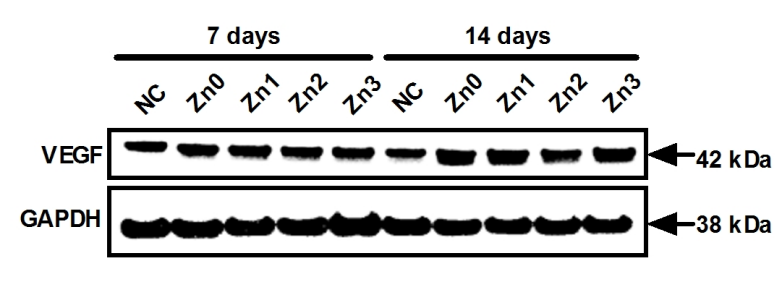

(E)

ON mRNA

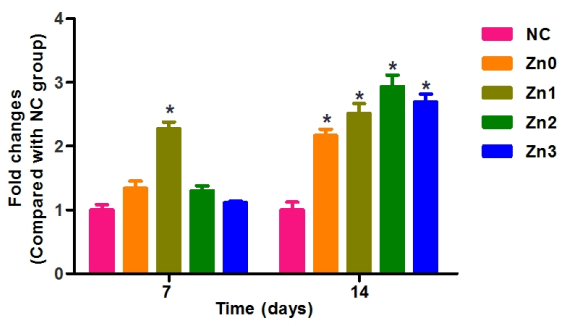

(G)

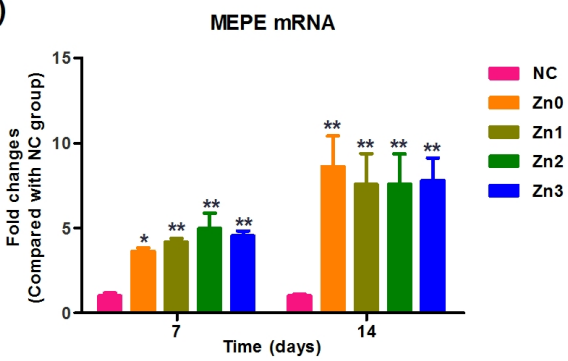

(I)

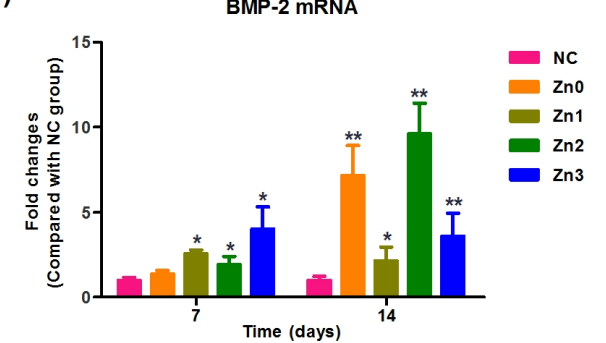

(K)

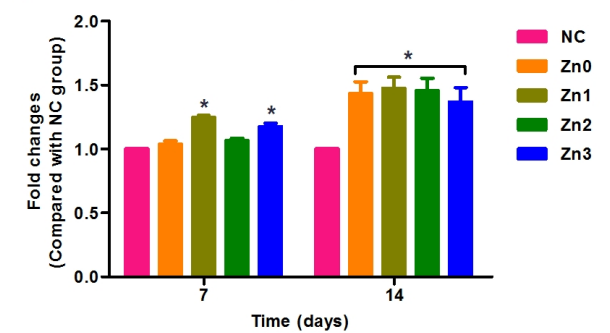

